EPJ Web of Conferences 82, 01001 (2015)

DOI: $10.1051 /$ epjconf/20158201001

(C) Owned by the authors, published by EDP Sciences, 2015

\title{
On the viscosity influence on a helical vortex flament evolution
}

\author{
M.V. Agafontseva ${ }^{2, a}$ and P.A. Kuibin ${ }^{1,2}$ \\ ${ }^{1}$ Novosibirsk State University, Russia \\ ${ }^{2}$ Kutateladze Institute of Thermophysics, Novosibirsk, Russia
}

\begin{abstract}
Helical vortices whose parameters have a strong influence on the efficiency of the apparatus is often occur in technical devices using swirling flow (cyclones, separators, etc.). To date the internal structure of such vortices is poorly understood. In [1] a model of helical vortex with uniform vorticity distribution in the core is proposed. Vortices arising in real flow always have a smooth vorticity distribution due to the viscosity action. The problem on steady moving helical vortices with the vortex core of small size in an inviscid fluid was solved in [2]. The non-orthogonal 'helical' coordinate system was introduced that allowed author to reduce the problem to two dimensional one. However, the velocity of the vortex motion was written only in the form of a quadratures computation of which is difficult. This paper presents first attempt for research on the diffusion and dynamics of a viscous helical vortex.
\end{abstract}

In this paper we consider the non-stationary problem of diffusion of helical vortex with a small diameter of core in viscous fluid. The aim is to describe the structure of the vortex and to find the speed of its movement.

Consider coordinate system introduced in [2]:

$$
\vec{x}=(\xi, \eta, \zeta)=\vec{H}(\xi)+\vec{N}(\xi) \eta+\vec{B}(\xi) \zeta .
$$

Here $\vec{H}(\xi)$ is a helix, unit vectors $(\vec{T}(\xi), \vec{N}(\xi), \vec{B}(\xi))$ are directed along the tangent, normal and binormal to the helix (components of some vector in this coordinate system will be shown by use angle brackets $\langle\circ, \circ, \circ>)$. Jacobian determinant of this system depends only on one coordinate: $J=1-k \eta$, $k=a /\left(a^{2}+l^{2}\right)$ is the curvature of helical line, $a$ is the helix radius and $2 \square l-$ is the helix pitch.

Helical symmetry condition [1] written in the Cartesian coordinates looks as follows:

$$
U_{z}=U_{0}+U_{x} \frac{y}{l}+U_{y} \frac{x}{l}, \quad \frac{\partial}{\partial z}=\frac{y}{l} \frac{\partial}{\partial x}-\frac{x}{l} \frac{\partial}{\partial y},
$$

$U_{0}$ means the axial component of velocity at the flow axis.

As for the velocity field $\langle u, v, w\rangle$, the symmetry condition brings to the form $J u-\tau \zeta v+\tau \eta w=$ $u_{0} l / \sqrt{a^{2}+l^{2}}, \tau-$ is the ratio of the helix torsion to its curvature.

\footnotetext{
${ }^{a}$ Corresponding author: m.agafontseva@gmail.com
}

This is an Open Access article distributed under the terms of the Creative Commons Attribution License 4.0, which permits unrestricted use, distribution, and reproduction in any medium, provided the original work is properly cited. 
The continuity equation takes the form [2]:

$$
\frac{\partial}{\partial \eta}(J v+\tau \zeta u)+\frac{\partial}{\partial \zeta}(J w-\tau \eta u)=0 .
$$

This equation allows introducing some specific stream function $\square$. The velocity components can be represented through the function $\square$ in view of the continuity equation and helical symmetry condition:

$$
\begin{gathered}
u=\left[\zeta \tau \frac{\partial \Psi}{\partial \zeta}-\eta \tau \frac{\partial \Psi}{\partial \eta}+\frac{\tau J U_{0}}{\sqrt{1+\tau^{2}}}\right] J^{-1}\left(J^{2}+\eta^{2} \tau^{2}+\zeta^{2} \tau^{2}\right)^{-1}, \\
v=\left[\left(J^{2}+\eta^{2} \tau^{2}\right) \frac{\partial \Psi}{\partial \zeta}+\eta \zeta \tau^{2} \frac{\partial \Psi}{\partial \eta}+\frac{\tau^{2} J \zeta U_{0}}{\sqrt{1+\tau^{2}}}\right] J^{-1}\left(J^{2}+\eta^{2} \tau^{2}+\zeta^{2} \tau^{2}\right)^{-1}, \\
w=\left[\left(J^{2}+\zeta^{2} \tau^{2}\right) \frac{\partial \Psi}{\partial \eta}+\eta \zeta \tau^{2} \frac{\partial \Psi}{\partial \zeta}+\frac{\tau^{2} J \eta U_{0}}{\sqrt{1+\tau^{2}}}\right] J^{-1}\left(J^{2}+\eta^{2} \tau^{2}+\zeta^{2} \tau^{2}\right)^{-1} .
\end{gathered}
$$

In [2] vorticity vector was introduced as $\vec{\omega}=f(\eta, \zeta)\langle 1,-\tau \zeta / J, \tau \eta / J\rangle$ and steady equation for the inviscid case was adduced. For the viscous formulation vorticity equation is:

$$
\frac{\partial}{\partial t} f(\eta, \zeta)+\left\{(J v+\tau \zeta u) \frac{\partial}{\partial \eta}+(J w-\tau \eta u) \frac{\partial}{\partial \zeta}\right\}\left\{\frac{f(\eta, \zeta)}{J}\right\}=v \Delta f(\eta, \zeta) .
$$

In order to determine the vorticity and stream function we use the second equation

$$
\omega=-\Delta \Psi
$$

where operator $\Delta$ reads

$$
\Delta=\frac{\left(\zeta^{2} \tau^{2}+J^{2}\right)}{J^{2}} \frac{\partial^{2}}{\partial \eta^{2}}+\frac{\left(\eta^{2} \tau^{2}+J^{2}\right)}{J^{2}} \frac{\partial^{2}}{\partial \zeta^{2}}-\frac{2 \tau^{2} \eta \zeta}{J^{2}} \frac{\partial^{2}}{\partial \eta \zeta}-\frac{\left(k J+\eta \tau^{2}\right)}{J^{2}} \frac{\partial}{\partial \eta}+\frac{\tau^{2} \zeta}{J^{2}} \frac{\partial^{2}}{\partial \eta \zeta} .
$$

For solving equation system (1)-(2) we use the two-scale decomposition method [3]. We set some nondimensional initial values of helical vortex and core radii: $a^{*}\left(t_{0}\right)=a_{0}, \varepsilon^{*}\left(t_{0}\right)=\varepsilon_{0}$, and consider two types of non-dimensional variables - external (marked with $*$ ) and internal (marked with line):

$$
\eta^{*}=\frac{\eta-a^{*}}{a^{*}}, \zeta^{*}=\frac{\zeta}{a^{*}}, \theta^{*}=\frac{a_{0}}{a^{*}}, \bar{\eta}=\frac{\eta-a^{*}}{\varepsilon^{*}}, \bar{\zeta}=\frac{\zeta}{\varepsilon^{*}}, \bar{\theta}=\frac{\varepsilon_{0}}{\varepsilon^{*}} .
$$

Required functions are represented as sums of two members, each of them depends on one type of the variables:

$$
\begin{gathered}
\frac{f\left(\eta, \zeta, t ; \delta_{0}\right)}{\omega_{0}}=f^{*}\left(\eta^{*}, \zeta^{*}, \theta^{*} ; \delta_{0}\right)+\frac{\bar{f}\left(\bar{\eta}, \bar{\zeta}, \bar{\theta} ; \delta_{0}\right)}{\delta_{0}^{2}}, \\
\frac{\Psi\left(\eta, \zeta, t ; \delta_{0}\right)}{\omega_{0} a^{* 2}}=\Psi^{*}\left(\eta^{*}, \zeta^{*}, \theta^{*} ; \delta_{0}\right)+\bar{\Psi}\left(\bar{\eta}, \bar{\zeta}, \bar{\theta} ; \delta_{0}\right) .
\end{gathered}
$$

$\omega_{0}$ means some typical scale of vorticity, $\delta_{0}=\varepsilon_{0} / a_{0}$ is the parameter of decomposition of the same order as $\delta=\varepsilon_{*} / a_{*}$. Equation system is divided into two systems: first is the internal problem equations (diffusion proper) and the second one is external problem equations (it coincides with the inviscid problem with additional members account for joining of internal and external solutions).

The zero order solution for vortex diffusion, which is valid for rectilinear as well as for any curved vortexes, is the Lamb-Oseen solution:

$$
f_{\tan }(\sigma, t)=\frac{\Gamma}{4 \pi \nu t} e^{-\sigma^{2} / 4 v t}
$$


$f_{\tan }$ means the vorticity projection on the tangent to the vortex axis. In our case $\sigma^{2}=\eta^{2}+\zeta^{2}$.

We obtained that after much time vorticity distribution beyond of vortex also takes the form of Lamb-Oseen distribution but depending of the radial coordinate $r=\sqrt{x^{2}+y^{2}}$.

Speed of motion of a helical vortex [1] as well as a vortex ring motion velocity [3] have a logarithmic dependence on vortex core radius and according to (6), at the initial stage of evolution it have a logarithmic dependence on time. Helical vortex movement is directed along a binormal to the helix. As a result, vortex rotates and moves forwardly. An impact to the binormal velocity produced by the finite size of vortex core and non-uniform distribution of vorticity in core looks as [1]:

$$
U_{b}^{i m p}=\frac{\Gamma k}{4 \pi}\left[\ln \frac{l}{\varepsilon}-\frac{1}{2}+4 \frac{\pi^{2}}{\Gamma^{2}} \int_{0}^{\varepsilon}\left(v^{2}+w^{2}\right) \sigma d \sigma\right] .
$$

In case of the uniform vorticity distribution integral member gives value 1/4. For distribution (6) one has

$$
U_{b}^{i m p}=\frac{\Gamma k}{4 \pi}\left[\ln \frac{l}{\sqrt{4 v t}}-\frac{1}{2}(1-\gamma+\ln 2)\right] .
$$

Here $\gamma \approx 0,5772$ is the Euler constant.

When considering the diffusion influence on the pressure pulsations induced by the helical vortex on the wall of a coaxial cylindrical pipe, it is necessary to take into account two effects. Growth of vortex core size initiates reduction of a pressure difference between maximum and minimum values in crosssection of pipe; amplitude of pressure difference is reduced. At the same time vortex radius increases. It means that vortex moves closer to the wall and initiates increasing of pulsations.

In the paper the first results on diffusion of a thin helical vortex at the high Reynolds numbers are obtained. In the future we plan to obtain the higher-order members for vorticity, stream function and helical vortex propagation velocity in the dependence of time. Also we plan to consider other limiting case - the case of low Reynolds numbers.

The work was supported by the Russian Scientific Fund (project No. 14-29-00093).

\section{References}

[1] S.V. Alekseenko, P.A. Kuibin, V.L. Okulov, S.I. Shtork, J. Fluid Mech. 382 (1999)

[2] A. Adebiyi, The Quarterly Journal of Mechanics and Applied Mathematics 34 (1979)

[3] A.A. Berezovskii, F.B. Kaplanskii, Fluid Dynamics 22 (1987) 